\title{
Assessing inflammatory status in COVID-19: a role in the pandemic?
}

\author{
Bernadette Corica ${ }^{1}$. Giulio Francesco Romiti ${ }^{1}$ (])
}

Received: 5 March 2021 / Accepted: 8 March 2021 / Published online: 26 March 2021

(c) Società Italiana di Medicina Interna (SIMI) 2021

Since the beginning of the COVID-19 pandemic in early 2020, several reports outlined the importance of the host inflammatory response in determining the severity of the disease and adverse outcomes in patients infected with SARSCoV-2 [1]. Pathological inflammation was postulated as one determinant of the disease outcomes [2], to the extent that a potential role for immunomodulating therapy was proposed and investigated, with the use of corticosteroids linked to reduced 28 day mortality in hospitalized patients [3]. However, differences in immune responses against COVID-19 may exist $[4,5]$, and the identification of patients with a dysregulated host response may be beneficial to improve prognostic assessment, especially in the first stages of the disease. In this scenario, several biomarkers (including $\mathrm{C}$ Reactive Protein (CRP), interleukins (IL), procalcitonin, and serum albumin) have been described and proposed as potentially useful to differentiate the severity of the disease [6-8].

In the article from Tyler Pitre and colleagues, the authors reported a retrospective, North-American cohort study on the association between several inflammatory biomarkerscommonly available in clinical practice-and outcomes in patients with COVID-19 [9]. Among 336 patients included in the analysis, CRP, serum albumin, and parameters derived from the white blood cells count [including the Neutrophil-Lymphocyte Ratio (NLR)] were associated with 28 day mortality after adjustment for age and sex. Moreover, a composite score based on CRP and serum albumin indicated a threefold higher risk of death for each point increase, with a maximum score of 2 .

The findings presented in this paper (although somewhat limited by missing data, the potential contribution of confounders such as bacterial superinfections, and the lack of a multivariate model combining several biomarkers) allow us to make some considerations, and offer insights on the

Giulio Francesco Romiti

giuliofrancesco.romiti@uniroma1.it

1 Department of Translational and Precision Medicine, Sapienza-University of Rome, Viale del Policlinico 155, 00161 Rome, Italy potential value of incorporating a simple assessment of the inflammatory status. Indeed, while the prognostic role of CRP was already outlined in this clinical scenario, being also included in the 4C Mortality Score [10], other tools that are used to stratify the risk of severe disease or death in COVID-19 patients still not include the assessment of the inflammatory status [11].

In this scenario, the study from Pitre et al. demonstrated how the evaluation of other, simple, and widely available inflammatory biomarkers might have a role in the prognostic stratification of COVID-19 patients. Among the biomarkers investigated, leukocytosis, lymphopenia, and several blood cell count ratios (including NLR) were associated with 28 day mortality. These results are consistent with findings already reported in the literature: NLR, which may reflect the balance between systemic inflammation and immunity, has been already described as a prognostic biomarker in other scenarios, including cardiovascular disease and cancer $[12,13]$. Consistently, a meta-analysis confirmed its association with mortality in patients with COVID-19 [14]; this also applies to lymphocyte count [15].

Beyond the traditional inflammatory markers, others have been postulated as useful predictors of poor outcomes in COVID-19 patients. In this study, reduced serum albumin levels were associated with 28 day mortality, consistently with what was observed in previous studies $[8,16]$ and confirmed by a recent meta-analysis [6]. Albumin has been linked with both anti-inflammatory anti-thrombotic effects [8], and low levels were found associated with the incidence of cardiovascular events [17], which also represent one leading cause of adverse events in patients with COVID-19 [18]. Taken together, these findings may suggest that low levels of albumin may predispose to poor prognosis, with multiple mechanisms; in this regard, a potential role of serum albumin administration for the treatment of COVID-19 has also been postulated [19].

The main implications of this study should be sought in the potential to improve our understanding of the disease and clinical course of patients with COVID-19. Although risk stratification of these patients is mainly based on clinical 
characteristics (i.e., age, comorbidities) and evaluation of hypoxemia (e.g., through assessment of oxygen saturation or $\mathrm{PaO}_{2} / \mathrm{FiO}_{2}$ ratio), there is probably room to improve our ability to anticipate the evolution of the disease by taking into account the inflammatory status. However, as for many other elements of this pandemic, there is plenty of uncertainties: we do not know which inflammatory marker offers the best prediction of poor outcomes among the ones available; probably, as demonstrated by the modified Glasgow Prognostic Score presented in this article, combining two or more variables would be useful. Although less sophisticated, the use of markers that are already widely available in clinical practice would promote a broader and more feasible assessment of inflammatory status in COVID-19 patients. In this direction, observational reports similar to that of Pitre and colleagues are very much needed to identify those biomarkers with better performances.

Further and more extensive studies are required to confirm these results and define limits of applicability, develop an integrated approach for assessing the inflammatory status along with clinical characteristics and severity of the disease, and finally evaluate if particular populations may require a different appraisal. In this scenario, systematic reviews and meta-analysis-which were indicated as potentially useful tools for the timely synthesis of evidence, and to answer urgent questions arising in this pandemic [20]—-may provide more solid ground for applying inflammatory biomarkers assessment in clinical practice. In our opinion, the evaluation of the inflammatory status may represent one promising approach in the management of COVID-19, not only to improve the prognostic stratification, but also to identify subgroups of patients who could benefit most from those treatments that target immune response.

\section{Declarations}

Conflict of interest The author(s) declare that they have no conflict of interest.

Statement of human and animal rights This article does not contain any studies with human participants or animals performed by any of the authors.

Informed consent None.

\section{References}

1. Mehta P, McAuley DF, Brown M et al (2020) COVID-19: consider cytokine storm syndromes and immunosuppression. Lancet 395:1033-1034. https://doi.org/10.1016/S0140-6736(20)30628-0

2. Merad M, Martin JC (2020) Pathological inflammation in patients with COVID-19: a key role for monocytes and macrophages. Nat Rev Immunol 20:355-362. https://doi.org/ 10.1038/s41577-020-0331-4

3. RECOVERY Collaborative Group, Horby P, Lim WS et al (2021) Dexamethasone in hospitalized patients with Covid-19. N Engl J Med 384:693-704. https://doi.org/10.1056/NEJMo a2021436

4. Arunachalam PS, Wimmers F, Mok CKP et al (2020) Systems biological assessment of immunity to mild versus severe COVID19 infection in humans. Science (80-) 369:1210-1220. https://doi. org/10.1126/SCIENCE.ABC6261

5. Carsetti R, Zaffina S, Piano Mortari E et al (2020) Different innate and adaptive immune responses to SARS-CoV-2 infection of asymptomatic, mild, and severe cases. Front Immunol. https:// doi.org/10.3389/fimmu.2020.610300

6. Paliogiannis P, Mangoni AA, Cangemi M et al (2021) Serum albumin concentrations are associated with disease severity and outcomes in coronavirus 19 disease (COVID-19): a systematic review and meta-analysis. Clin Exp Med. https://doi.org/10.1007/ s10238-021-00686-Z

7. Zeng F, Huang Y, Guo Y et al (2020) Association of inflammatory markers with the severity of COVID-19: a meta-analysis. Int $\mathbf{J}$ Infect Dis 96:467-474. https://doi.org/10.1016/j.ijid.2020.05.055

8. Violi F, Cangemi R, Romiti GF et al (2020) Is albumin predictor of mortality in COVID-19? Antioxid Redox Signal. https://doi. org/10.1089/ars.2020.8142

9. Pitre T, Jones A, Su J et al (2021) Inflammatory biomarkers as independent prognosticators of 28-day mortality for COVID-19 patients admitted to general medicine or ICU wards: a retrospective cohort study. Intern Emerg Med. https://doi.org/10.1007/ s11739-021-02637-8

10. Knight SR, Ho A, Pius R et al (2020) Risk stratification of patients admitted to hospital with covid-19 using the ISARIC WHO clinical characterisation protocol: development and validation of the 4C mortality score. BMJ 370:22. https://doi.org/10.1136/bmj. m3339

11. Artero A, Madrazo M, Fernández-Garcés M et al (2021) Severity scores in COVID-19 pneumonia: a multicenter, retrospective, cohort study. J Gen Intern Med. https://doi.org/10.1007/ s11606-021-06626-7

12. Cupp MA, Cariolou M, Tzoulaki I et al (2020) Neutrophil to lymphocyte ratio and cancer prognosis: an umbrella review of systematic reviews and meta-analyses of observational studies. BMC Med 18:360. https://doi.org/10.1186/s12916-020-01817-1

13. Angkananard T, Anothaisintawee T, McEvoy M et al (2018) Neutrophil lymphocyte ratio and cardiovascular disease risk: a systematic review and meta-analysis. Biomed Res Int. https://doi. org/10.1155/2018/2703518

14. Li X, Liu C, Mao Z et al (2020) Predictive values of neutrophil-tolymphocyte ratio on disease severity and mortality in COVID-19 patients: a systematic review and meta-analysis. Crit Care. https:// doi.org/10.1186/s13054-020-03374-8

15. Wagner J, DuPont A, Larson S et al (2020) Absolute lymphocyte count is a prognostic marker in Covid-19: a retrospective cohort review. Int J Lab Hematol 42:761-765. https://doi.org/10.1111/ ijlh. 13288

16. Huang J, Cheng A, Kumar R et al (2020) Hypoalbuminemia predicts the outcome of COVID-19 independent of age and comorbidity. J Med Virol 92:2152-2158. https://doi.org/10.1002/ jmv.26003

17. Ronit A, Kirkegaard-Klitbo DM, Dohlmann TL et al (2020) Plasma albumin and incident cardiovascular disease results from the CGPS and an updated meta-analysis. ArteriosclerThrombVascBiol 40:473-482. https://doi.org/10.1161/ATVBAHA.119. 313681

18. Nishiga M, Wang DW, Han Y et al (2020) COVID-19 and cardiovascular disease: from basic mechanisms to clinical 
perspectives. Nat Rev Cardiol 17:543-558. https://doi.org/10. 1038/s41569-020-0413-9

19. The potential use of albumin in COVID-19 patients I The BMJ. https://www.bmj.com/content/368/bmj.m810/rr-41. Accessed 16 Feb 2021

20. Romiti GF, Corica B, Cangemi R et al (2020) Need for innovative and timely synthesis of evidence during Covid-19 outbreak. Eur J Intern Med 77:165-166. https://doi.org/10.1016/j.ejim.2020.06. 007
Publisher's Note Springer Nature remains neutral with regard to jurisdictional claims in published maps and institutional affiliations. 